\title{
Chimeric antigen receptor $\mathrm{T}$ cell (CAR-T) immunotherapy for solid tumors: lessons learned and strategies for moving forward
}

\author{
Jian $\mathrm{Li}^{1+}$, Wenwen $\mathrm{Li}^{2+}$, Kejia Huang ${ }^{3}$, Yang Zhang ${ }^{3}$, Gary Kupfer ${ }^{4}$ and Qi Zhao ${ }^{5^{*}}$
}

\begin{abstract}
Recently, the US Food and Drug Administration (FDA) approved the first chimeric antigen receptor T cell (CAR-T) therapy for the treatment CD19-positive B cell acute lymphoblastic leukemia. While CAR-T has achieved remarkable success in the treatment of hematopoietic malignancies, whether it can benefit solid tumor patients to the same extent is still uncertain. Even though hundreds of clinical trials are undergoing exploring a variety of tumor-associated antigens (TAA), no such antigen with comparable properties like CD19 has yet been identified regarding solid tumors CAR-T immunotherapy. Inefficient T cell trafficking, immunosuppressive tumor microenvironment, suboptimal antigen recognition specificity, and lack of safety control are currently considered as the main obstacles in solid tumor CAR-T therapy. Here, we reviewed the solid tumor CAR-T clinical trials, emphasizing the studies with published results. We further discussed the challenges that CAR-T is facing for solid tumor treatment and proposed potential strategies to improve the efficacy of CAR-T as promising immunotherapy.
\end{abstract}

Keywords: CAR-T, Solid tumor, Tumor microenvironment, Antigen recognition specificity, Safety control

\section{Background}

Recently, the US Food and Drug Administration (FDA) approved the first chimeric antigen receptor $\mathrm{T}$ cell (CAR-T) therapy for the treatment of children and young adults with relapsed or refractory B cell acute lymphoblastic leukemia (ALL) positive for CD19 antigen [1, 2]. Chimeric antigen receptors (CARs) are chimeric immunoglobulin $\mathrm{T}$ cell receptor (TCR) molecules derived from transgenes encoding for single-chain variable fragments $(\mathrm{scFv})$, which originate from antibodies capable of recognizing tumor-associated antigens (TAA) $[3,4]$. Mechanistically, the CAR-T cell recognizes and binds to TAA, inducing a conformational change that transmits the binding signal into the CAR-T cell. Activation signal through the $\mathrm{CD} 3 \zeta$ domain and costimulatory domains activate CAR-T cell, leading to cytokine release and transcription factor expression, which promote $T$ cell survival and function and eventually induce cytotoxic activities against tumor cells $[5,6]$. The molecular

\footnotetext{
* Correspondence: zhaoqi@cdu.edu.cn

${ }^{\dagger}$ Equal contributors

${ }^{5}$ College of Pharmacy and Biological Engineering, Chengdu University,

Chengdu 610106, China

Full list of author information is available at the end of the article
}

mechanisms of CAR-T immunotherapy were summarized in Fig. 1.

The designs of CARs are grouped schematically into three generations with increasing costimulatory activity [7]. The first generation CARs are conjugated with TCR-CD3 $\zeta$ chain alone, which is capable of providing a comparable stimulatory signal to that of the entire CD3 complex $[8,9]$. However, this CAR configuration is insufficient to prime resting $\mathrm{T}$ cells for proliferation and cytokine production, affecting sustained antitumor responses in vivo [10]. With the aim to enhance the stimulation effect, the secondgeneration CARs include a costimulatory module on the basis of the first generation, which was initially designed in the 1990s $[8,9,11,12]$. CD28 is one of the most commonly utilized molecules for this purpose to promote interleukin2 (IL-2) secretion and improve T cell activity [13-16]. On top of $\mathrm{CD} 3 \zeta$ and co-stimulators like CD28, additional costimulatory domains, such as OX40 or 4-1BB, were added to the third generation CARs to further enhance the signaling capacity $[17,18]$. The fourth-generation CARs added IL-12 to the base of the second-generation constructs, which are known as $\mathrm{T}$ cell redirected for universal cytokine-mediated killing (TRUCKs). TRUCKs augment T cell activation and 

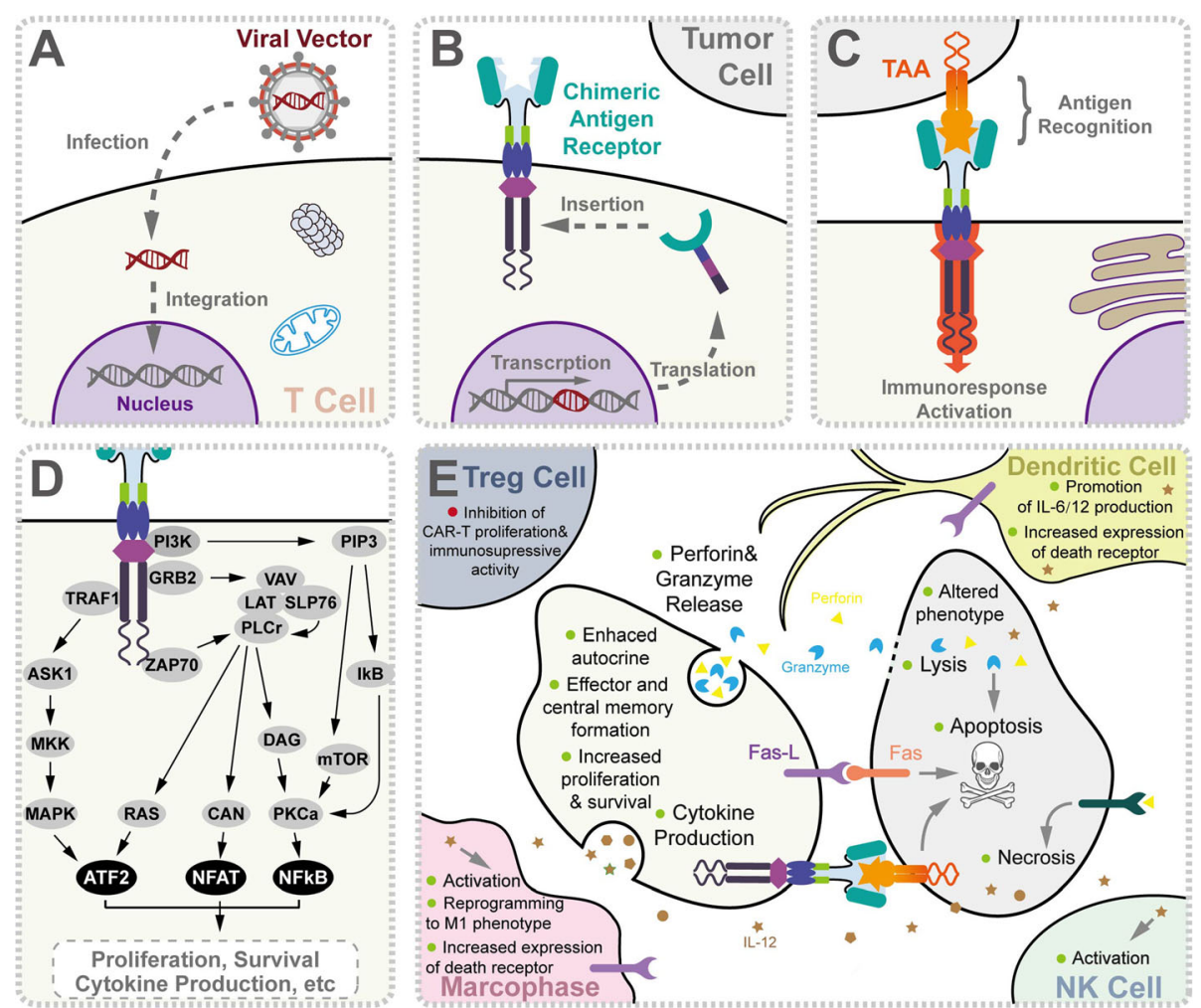

Fig. 1 Molecular mechanism of chimeric antigen receptor T cell-mediated antitumor activity. a The chimeric T cell receptor coding sequence is delivered by viral vector. After entering into T cells (beige), virus was uncoated and transgene was preferably integrated at genome transcriptional start sites using specific vector designs, such as MLV retrovirus and piggyBac transposon. b CAR transgenes were endogenously transcript by host machinery, translated, and later inserted onto the T cell surface. c Association of CARs to TAA (orange) on tumor cell surface activates T cell for immunological response, for example, signaling network of CAR-T composed of CD8-CD28-CD137-CD3Z domains was shown in (d). CAR-T-mediated immune response was reported to be amplified by ZAP70, TRAF1, PI3K, and GRB2 as well as other uncharacterized factors, giving rise to upregulation of signaling intermediates and subsequent pro-death gene transcriptions. e Upon CAR activation, T cells secreted cytokines (brown), perforins (bright yellow), and granzymes (blue) as well as activated death receptors, which triggered downstream targets. These subcellular events directly or subsequently contribute to specific death of tumor cells, including perforin and granzyme release, cytokine production, direct lysis, apoptosis, necrosis, reprogrammed phenotype, and immuno-memory formation in T cells, tumor cells (gray), macrophages (pink) (via IL-6, IL-10, IL-12,

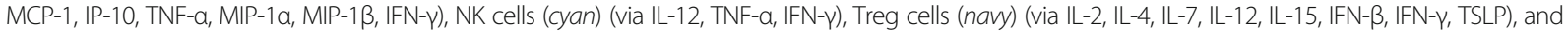
dendritic cells (yellow) (via IL-6, IL-10, IL-12, TNF-a, MIP-1a, MIP-1 $\beta$, IFN- $\gamma$ ). Abbreviation: NK cells natural killer cells, TAA tumor-associated antigen, Treg cells regulatory $T$ cells

activate and attract innate immune cells to eliminate antigen-negative cancer cells in the targeted lesion. Such TRUCK T cells can also treat viral infections, metabolic disorders, and auto-immune diseases [19-21].

Whereas ongoing CAR-T clinical trials for the treatment of leukemia and lymphoma have demonstrated durable remission of the disease or even cure, CAR-T therapy targeting solid tumor is still in an infant stage. One of the most frequently asked questions is whether CAR-T can benefit solid tumor patients to the same extent as it does for blood malignancies. Here, we reviewed the published results of clinical studies for solid tumor CAR-T treatment. We further discussed the challenges that CAR-T is facing for solid tumor treatment and proposed potential strategies to improve the efficacy of CAR-T as promising immunotherapy.

\section{Clinical trials using engineered CAR-T cells to treat solid tumor}

Because of the success achieved in CAR-T therapy targeting $B$ cell malignancies and the advancements in CAR-T preclinical studies for solid tumors, more than 100 CAR-T clinical trials targeting solid tumors have been initiated at medical centers all over the world (Table 1).

\section{Tumor-associated antigens and CAR design}

So far, no such cell surface antigen with comparable properties as CD19 has yet been identified regarding solid tumors. An ideal molecule for CAR targeting should be overexpressed on cancer cell surface of many patients, with zero or very low expression in normal tissues. Currently, TAAs, including mesothelin (MSLN), 


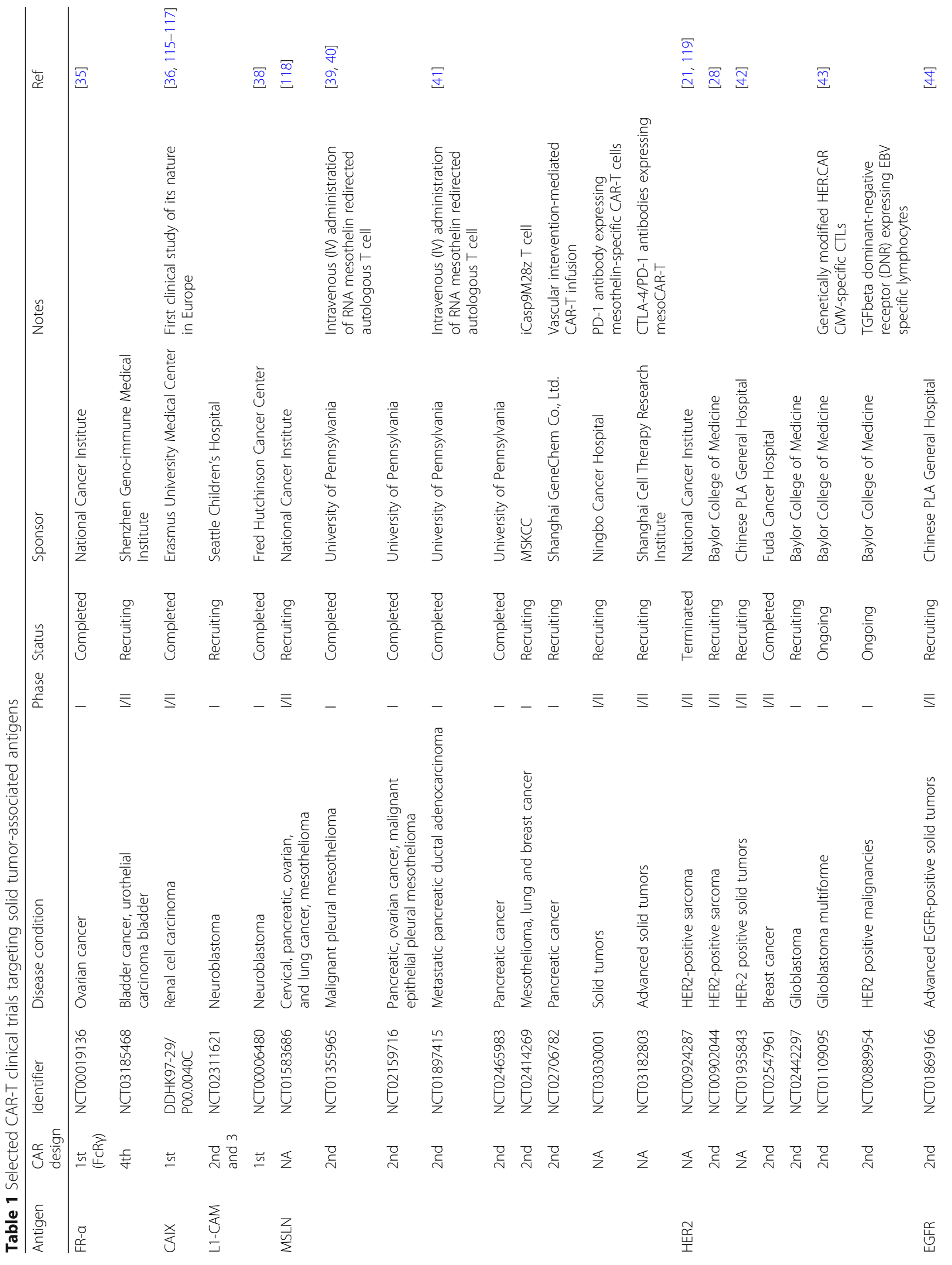




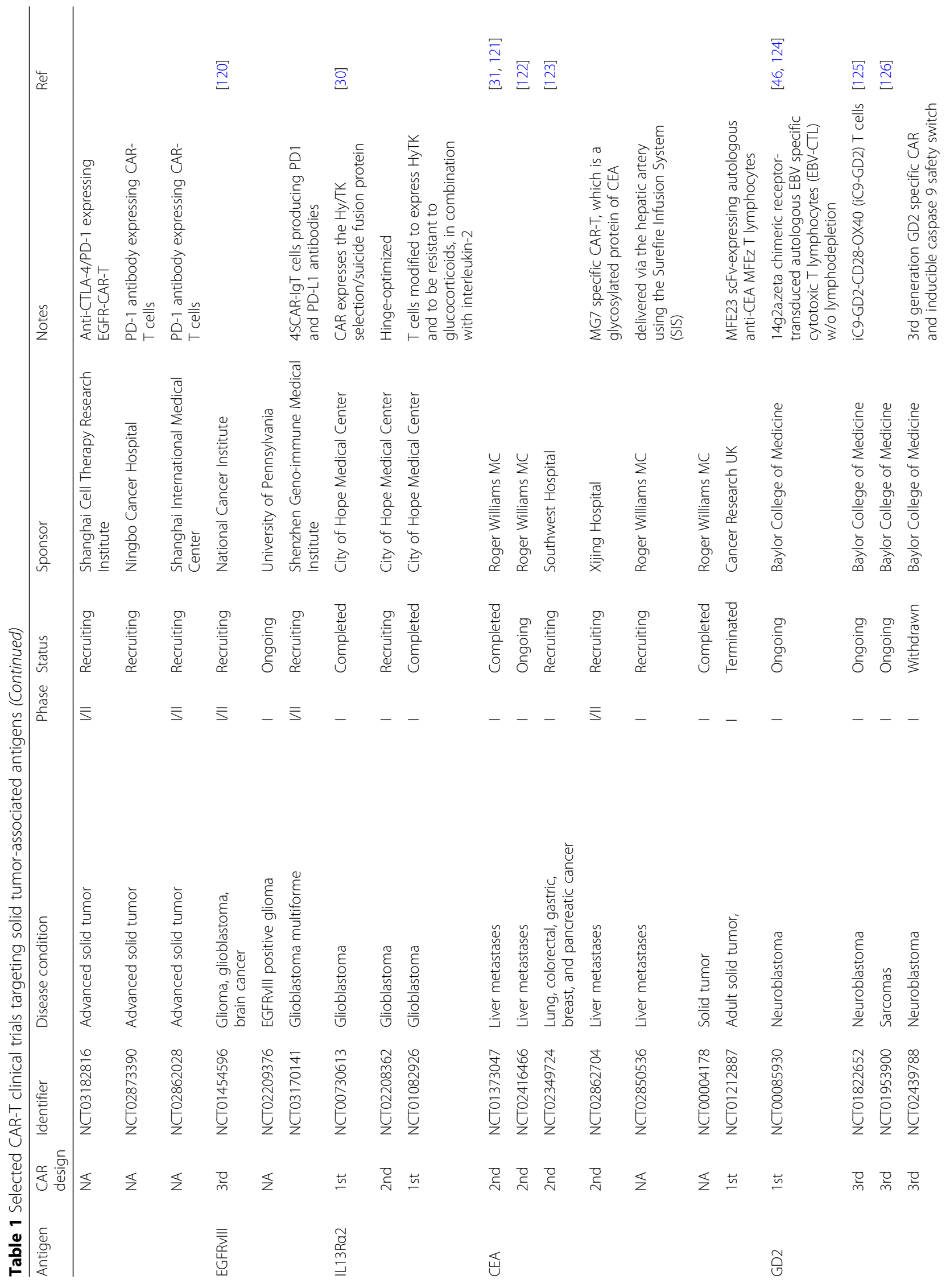




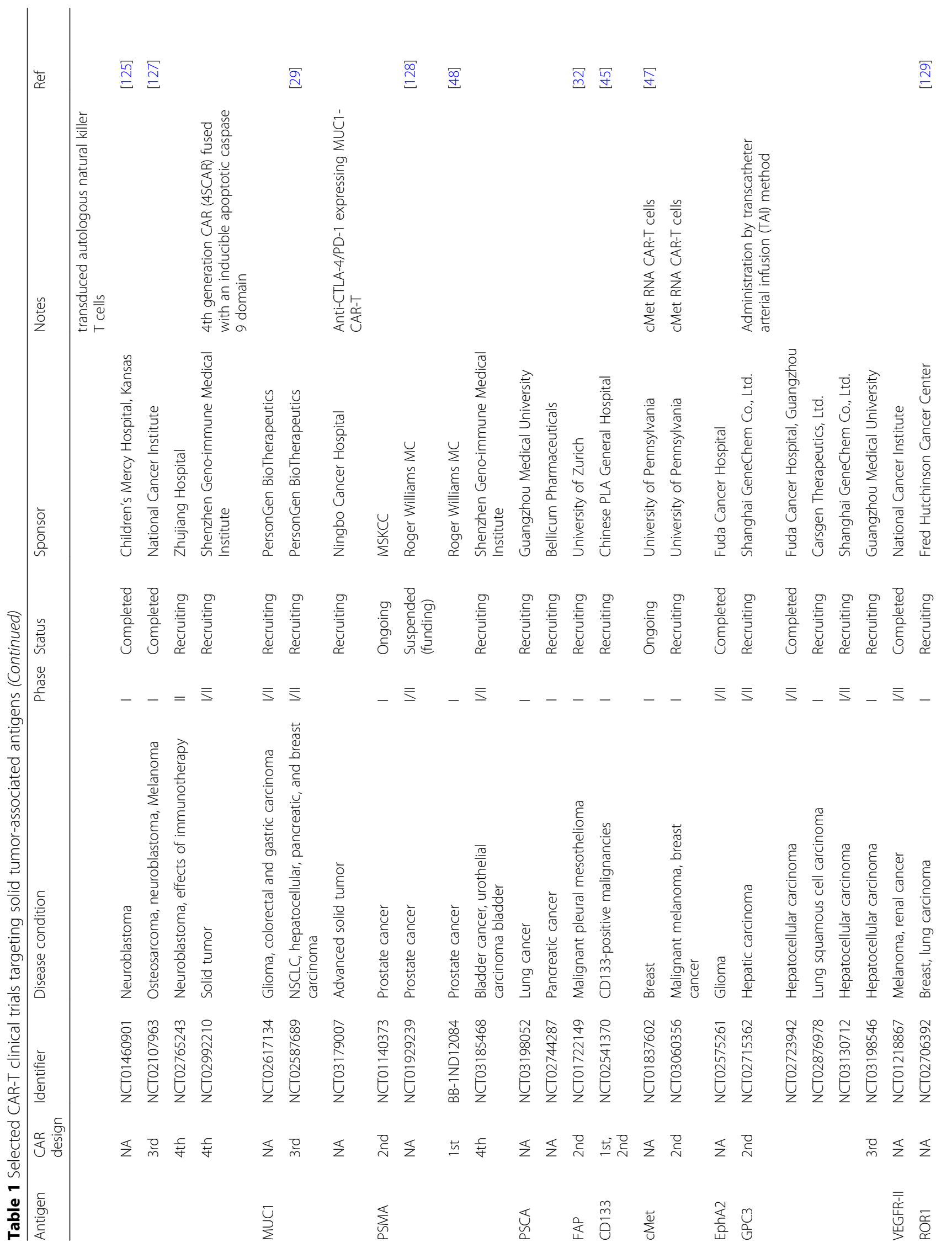




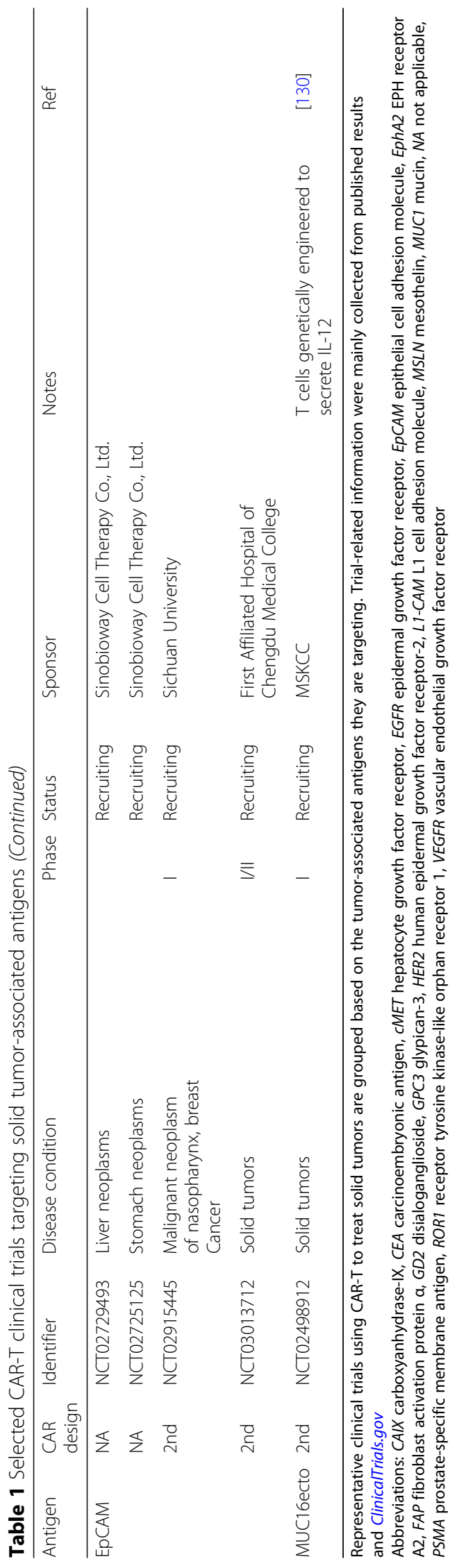


HER2, EGFR/EGFRvIII, GD2, CEA, IL13R $\alpha 2$, MUC1, FAP, PSMA, and PSCA, are extensively investigated in CAR-T therapy for solid tumors [22, 23]. TAAs currently being exploited for CAR-T therapy in solid tumors are summarized (Fig. 2). Yu and colleagues comprehensively discussed these antigens regarding their biological functions and antitumor activities [22]. As shown in Table 1, most of the solid tumor CAR-T clinical trials utilize the second or third generation of CARs, which contain either CD28 alone or CD28-4-BB1/OX40 as the costimulatory signal. Notably, a few of these studies, e.g., the trials targeting GD2 (NCT02765243, NCT02992210), PMSA (NCT03185468), FR- $\alpha$ (NCT03185468), investigated the efficacy of the fourth-generation CARs, i.e., TRUCK, which includes a transgenic cytokine expression cassette in the CAR constructs [24]. Because of the tremendous phenotypic diversity in solid tumor lesions, a reasonable number of cancer cells are not recognized by a given CAR. The introduction of a transgenic cytokine such as interleukin-12 (IL-12) initiates universal cytokine-mediated killing towards those cancer cells that are invisible to CAR-T cells [19].

CAR-T cell therapy must carefully balance efficient $T$ cell activation, to ensure antitumor activity, with the

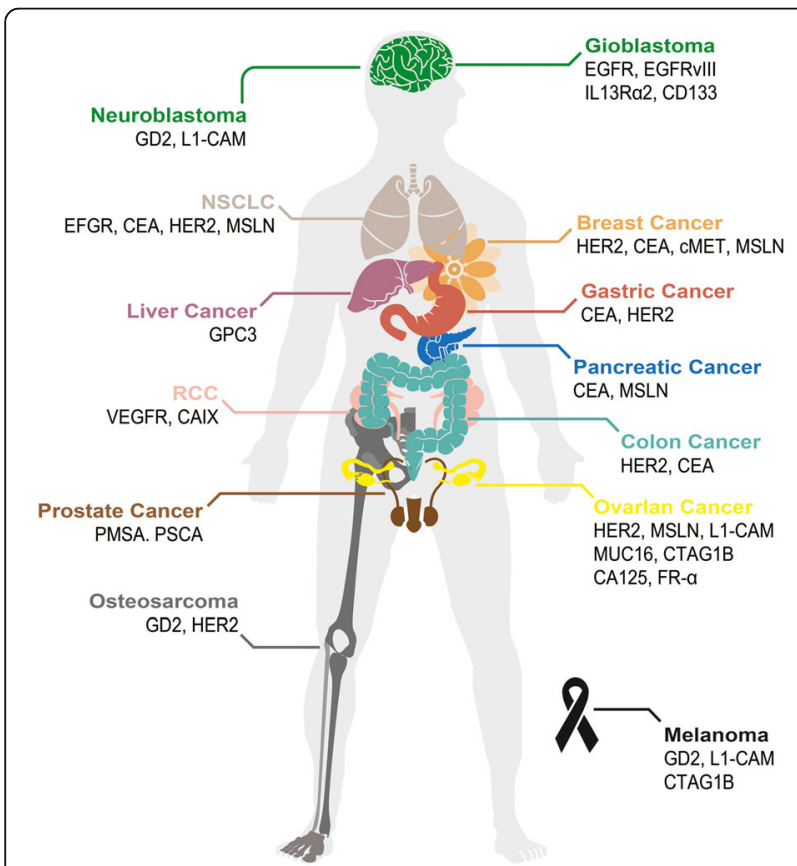

Fig. 2 Tumor-associated antigens targeted in CAR-T therapy. Schematic illustration of a human body whose tissues or organs have been investigated in preclinical and clinical studies for solid tumor immunotherapy using CAR-T, including brain (green), lungs (beige), mammary gland (orange), liver (purple), stomach (red), pancreas (blue), kidneys (pink), colon (cyan), male reproductive system (brown), ovary (yellow), and bones (gray) as well as skin (black). Abbreviation: CCA cholangiocarcinoma, MPM malignant pleural mesothelioma, NSCLC non-small-cell lung carcinoma, RCC renal cell carcinoma potential for uncontrolled cytotoxicity. Some recent clinical trials included an inducible caspase 9 (iCasp9) "safety switch" in their CAR construct, which allows for the removal of inappropriately activated CAR-T cells $[25,26]$. The administration of the small molecule drug AP1903 causes the dimerization and activation of iCasp9, resulting in rapid induction of apoptosis in transduced cells. Two CAR-T clinical trials targeting GD2 performed at Baylor College of Medicine incorporated such iCasp9 switch into their third generation CAR constructs (NCT01822652, NCT02439788). In another recently initiated trial led by Chang, a fourthgeneration CAR was fused with the iCasp9 domain (NCT02992210).

PD-L1 expression in solid tumors counteracts the efficacy of CAR-T cell [27]. To overcome the inhibitory effect of PD-LI expression on CAR-T therapy, some clinical trials (e.g., NCT03030001, NCT03182803-MSLN, NCT03182816, NCT02873390, NCT02862028-EGFR, NCT03170141-EGFRvIII, and NCT03179007-MUC1) add PD-1 dominant-negative receptor expressing gene to CAR-T cells, providing cell-intrinsic checkpoint blockade and increase antitumor efficacy. Based on the currently available data, anti-PD-1 combination therapy approach may be useful to augment CAR-T cell efficacy and persistence in patients [27].

\section{T cell dosage, administration, and persistence}

According to the published results and clinical trial description available on the website, the majority of the CAR-T clinical trials use a dose escalation regime, which usually covers two log steps with a starting point ranged from around $1 \times 10^{6}$ and $1 \times 10^{9}$ CAR-T cells [24]. It is important to keep in mind that the percentage of CARpositive $T$ cells varies significantly not only among different trials but also among different batches within single trial [24]. One of the major sources for such variation is the different production procedure of CAR-T cells adopted by different trials, emphasizing the importance of standardizing the CAR-T cells production protocol. Notably, to increase the tolerability of the treatment and to lower the risk of side effects, the given CAR-T cell dose is often split over multiple injections. For example, Ahmed and colleagues reported administration of $1 \times$ $10^{4}-1 \times 10^{8} / \mathrm{m} 2$ HER2 specific CAR-T cells in up to 9 infusions (NCT00902044) [28]. Typically, CAR-T cells are infused intravenously. However, intratumoral (NCT02587689) [29], intracranial (NCT00730613) [30], hepatic artery (NCT01373047) [31], and pleural [32] administration are being investigated as well. The CAR-T cell administration method could have a dramatic impact on the efficacy of the treatment. Brown et al. compared the effectiveness of two intracranial CAR-T cell delivery route-infusion into the resected tumor 
cavity and infusion into the ventricular system [33]. While intracavitary therapy appeared to control local tumor recurrence, progression of glioblastoma at distant sites was observed. By contrast, intraventricular administration of CAR-T cells leads to the regression of all central nervous system tumors, including spinal tumors. After the administration, CAR-T cell persistence is a key factor that determines the efficacy of the therapy. One major problem of current CAR-T cell immunotherapy is that $\mathrm{T}$ lymphocytes have limited replicative lifespans [34], which potentially influences the long-term antitumor effect of CAR-T cell immunotherapy. The reported $T$ cell persistence in published clinical trials ranged from up to 4 weeks (DDHK97-29/P00.0040C, BB-IND 12084) to up to 192 weeks (NCT00085930).

\section{Clinical outcomes and toxicities}

Some early attempts using CAR-T to treat solid tumors were not successful. In 2006, Kershaw and colleagues tested the efficacy of CAR-T cells targeting ovarian cancer cells expressing alpha-folate receptor (FR- $\alpha$ ) (NCT00019136) [35]. Even though the patients can tolerate the administration of FR- $\alpha$ specific CAR-T cells, no clinical response was observed in all the patients (14 out of 14), likely because of the inefficient localization and short-term persistence of the CAR-T cells [35]. Lamers and colleagues treated 12 patients with CAIX-expressing metastatic renal cell carcinoma with CAIX specific CAR-T cells [36, 37]. Unfortunately, no clinical responses were observed, and some patients developed anti-CAR-T cell antibodies and severe live enzyme disturbance.

Park et al. reported treating metastatic neuroblastoma with L1-CAM specific CAR-T cells at a dosage of $1 \times 10^{8}$ cells $/ \mathrm{m}^{2}$ to $1 \times 10^{9}$ cells $/ \mathrm{m}^{2}$ (NCT00006480) [38]. The persistence of cells was measured to be 1-7 days for patients with heavy disease burden and 42 days for a patient with light disease burden. One of the six enrolled patients achieved stable disease after treatment [38]. A series of CAR-T clinical trials targeting MSLN have been performed in many different types of solid tumors, such as mesothelioma (NCT01355965), pancreatic cancer (NCT01583686, NCT02465983, NCT02706782), and breast cancer (NCT02792114). In one of these trails, advanced mesothelioma patients were administered autologous $\mathrm{T}$ cells electroporated with the mRNA encoding for MSLN CAR (NCT01355965) [39, 40]. Moderate clinical responses were observed as supported by the detection of MSLN specific CAR-T cells in the tumor site and the transient elevation of inflammatory cytokines [39, 40]. MSLN CAR T cell infusions were well tolerated at the dosage tested without severe toxicities $[39,40]$. Unfortunately, one of the study subjects was reported to develop severe anaphylaxis and cardiac arrest after the third infusion of MSLN CAR-T cells [40], which is likely to result from the inclusion of the murine $\mathrm{SS} 1 \mathrm{scFv}$ in the CAR-T design. In another trial for pancreatic ductal adenocarcinoma (PDAC) (NCT01897415), Beatty and colleagues utilized mRNA-encoded mesothelin-specific CAR to treat six patients, in which the infusion were well tolerated and preliminary evidence of antitumor efficacy was observed, supported by stable disease seen in two treated patients [41].

Clinically, the safety and efficacy of HER2-specific CAR$\mathrm{T}$ cells in patients with relapsed/refractory HER2-positive sarcoma has been evaluated in a phase I/II clinical study (NCT00902044, NCT01109095, and NCT00924287). In a dose escalating trial (NCT00902044, $1 \times 10^{4} / \mathrm{m}^{2}$ to $1 \times$ $\left.10^{8} / \mathrm{m}^{2}\right), 4$ out of 19 subjects acquired stable disease [28]. Feng et al. performed a phase I clinical trial investigating HER2-specific CAR-T cells in patients with advanced biliary tract cancers (BTCs) and pancreatic cancers (PCs) (NCT01935843). Among the 6 patients received HER2specific CAR-T infusion, one patient obtained a 4.5month partial response and the other 5 patients achieved stable disease with mild to moderate adverse events [42]. In another trial (NCT01109095), 17 CMV-seropositive patients with radiologically progressive HER2-positive glioblastoma were infused with HER2 CMV bispecific CAR-T cells at the dose of $1 \times 10^{6} / \mathrm{m}^{2}-1 \times 10^{8} / \mathrm{m}^{2}$ [43] Such treatments were well tolerated without severe adverse events or cytokine release syndrome, and 7 out the 17 treated patients achieved stable disease [43].

Feng and colleagues reported the clinical trials of EGFR specific CAR-T treating non-small cell lung cancer (NCT01869166) [44]. Five out of the 11 treated patients achieved stable disease and 2 achieved partial response. The CAR-T infusion-related adverse events were mild and manageable. Recently, Feng et al. reported treating a patient diagnosed as advanced unresectable/metastatic cholangiocarcinoma (CCA) with CAR-T cocktail immunotherapy, which was composed of successive infusions of CAR-T cells targeting epidermal growth factor receptor (EGFR) and CD133 (NCT01869166, NCT02541370) [45]. The patient achieved an 8.5-month partial response (PR) from the CART-EGFR therapy and a 4.5-month-lasting PR from the CART133 treatment. However, a series of adverse events were also observed in the course of treatment, including deteriorative grade 3 systemic subcutaneous hemorrhages and congestive rashes together with serum cytokine release.

In 2015, Brown et al. reported that three patients with recurrent glioblastoma were treated with CAR-T cells targeting IL13R $\alpha 2$ (NCT00730613) [30]. Patients received up to 12 local infusions at a maximum dose of $1 \times 10^{8}$ CAR-T cells. Evidence for transient antitumor activity was observed in two of the patients with 
manageable temporary central nerve system inflammation. The same group conducted another clinical trial (NCT02208362), in which tumor regression and increased production of cytokines and immune cells were observed [33]. Encouragingly, the clinical response continued for 7.5 months after the initiation of CAR-T cell therapy, indicating a relatively long persistence of CAR$\mathrm{T}$ cells.

Katz and colleagues conducted a phase I trial to test CAR-T in patients with CEA-positive liver metastases (NCT01373047). Among the six patients who completed the protocol, one patient remained alive with stable disease at 23 months following CAR-T treatment and five patients died of progressive disease [31]. Biopsies demonstrated an increase in liver metastases necrosis or fibrosis in four out of six patients. No patient suffered a grade 3 or 4 adverse event related to the CAR-T treatment. Louis and colleagues evaluated the efficacy of GD2-specific CAR-T in 19 patients either with remission (8 patients) or progressive neuroblastoma (11 patients) (NCT00085930) [46]. Three of 11 patients with active disease achieved complete remission and up to 192 weeks of CAR-T cell persistence was observed in the trial [46].

Clinical trials investigating the efficacy of CAR-T cells targeting MUC1 (NCT02587689) [29], cMet (NCT01837602, NCT03060356) [47], PSMA (BB-IND 12084) [48], VEGFR2 (NCT01218867) have been reported. Relevant information and results of CAR-T clinical trials were summarized in Table 2.

As learned from the results of these clinical trials, CAR-T cells face a unique set of challenges in the case of solid tumors. Some of the issues appear to be the absence of unique tumor-associated antigens, the inefficient homing of $\mathrm{T}$ cells to tumor sites, and the limited persistence of CAR-T cells. Moreover, the immunosuppressive microenvironment within the tumor tends to inhibit CAR-T cell function strongly. While a seemingly complicated, fulfilling all of above requirements can be accomplished efficiently through both intrinsic and/or extrinsic modifications of CAR-T cells.

\section{Overcoming challenges with smarter CAR designs}

Different from $B$ cell malignancies, the application of the CAR-T cell strategy to non-hematopoietic cancer is faced with physical barriers as well as a variety of approaches that tumors employ to blunt host immunesurveillance [49]. Therefore, obstacle factors existing in current CAR-T trials such as fibrosis, inflammation, autoimmunity, $\mathrm{T}$ cell exhaustion and persistence, or recurrence must be overcome with smarter redirected $\mathrm{T}$ cell designs to achieve optimal therapeutic results (Fig. 3).

\section{Enhancing trafficking}

Tumors are organized tissues with numerous connections with surrounding cells [50], including resident immune system, i.e., myeloid compartment and lymphoid compartment [51], vasculature, fibroblasts, signaling molecules, and the extracellular matrix [52]. In addition, the low $\mathrm{pH}$, low oxygen, low nutrients condition, and the tumor surrounding tissues, especially blood vessels and fibroblasts, make therapeutic delivery cruelly difficult [53].

In current trials, homing of CAR-T cells to the tumor sites is often inefficient owing to the reasons mentioned above. The successful application of CAR-T cells against hematological malignancies is at least partially attributed to the fact that tumor and effector $\mathrm{T}$ cells tended to migrate to similar sites due to their shared hematopoietic origins. Solid tumors are known to secrete immuno-factors, including chemokine, cytokine, and growth factors, preventing effector $\mathrm{T}$ cells from infiltrating into the tumor bed (Fig. 3a). In 2010, a recombinant chemokine receptor ligand CCR2(b) was introduced into CAR-T cells targeting MSLN [54], and GD2 [55], because the endogenous chemokine receptors on $\mathrm{T}$ cells adequately mismatch with the chemokines secreted by the tumors. Beyond minimizing the effects of chemokine, strategies targeting activated surrounding fibroblast (fibroblast activation protein, FAP) in mouse models of mesothelioma and lung cancer [56, 57], vasculature (anti-VEGFR-2) in melanoma, and renal cancer (NCT01218867) [58], as well as tumor stroma (heparanase) in neuroblastoma mice [59], have been tested.

Insufficient $\mathrm{T}$ cell trafficking is a major functional challenge in anti-cancer immunotherapy. Little has been improved regarding $\mathrm{T}$ cell infiltration in the past decade, which is primarily due to our limited understanding of the tumor surrounding compartments and their effects on CAR-T cells [51]. More tumor-associated biomarkers related to $\mathrm{T}$ cell filtration should be investigated, such as alpha smooth muscle actin, Thy-1, desmin, and S100A4 protein [60]. In addition, strategies developed in nonCAR-T therapy context might also be beneficial to CAR$\mathrm{T}$ design and utilized for penetrating the physical barrier and thus enhancing CAR-T cell trafficking. Beyer et al. described a self-dimerizing epithelial intercellular junction opener JO-1 that bound to desmoglein 2 [61]. Manipulation of JO-1 transiently broke tight junctions in polarized epithelial tumor cells, resulting in increased efficiency in mAb treatments (trastuzumab and cetuxi$\mathrm{mab})$ in xenograft tumor models. Moreover, intratumoral relaxin expression was reported to degrade extracellular matrix protein around solid tumors transiently, which enhanced trastuzumab treatment [62] (Fig. 3b). Non-signaling extracellular hinge domain of CAR-T has also been shown to exhibit an impact on the 


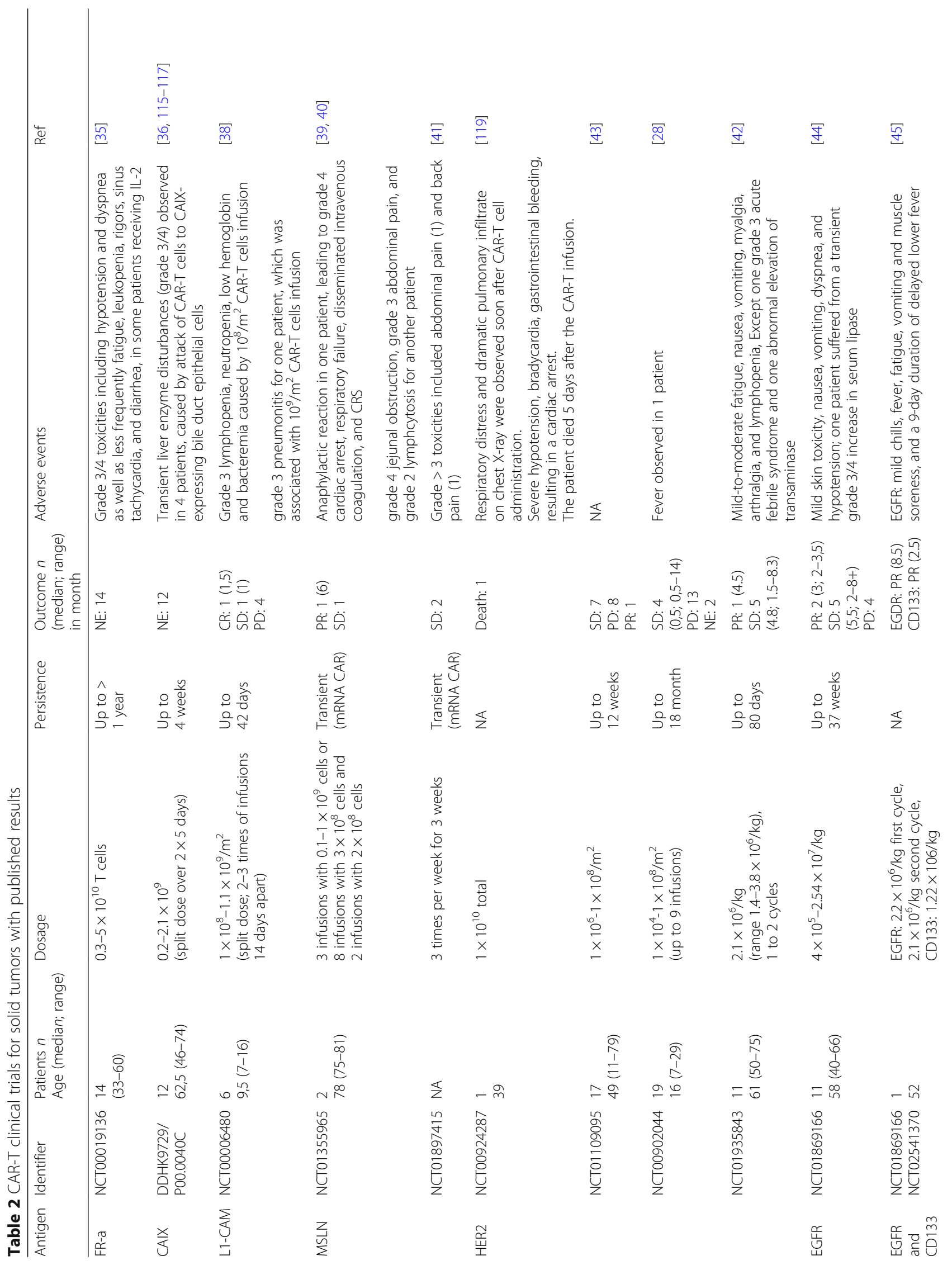




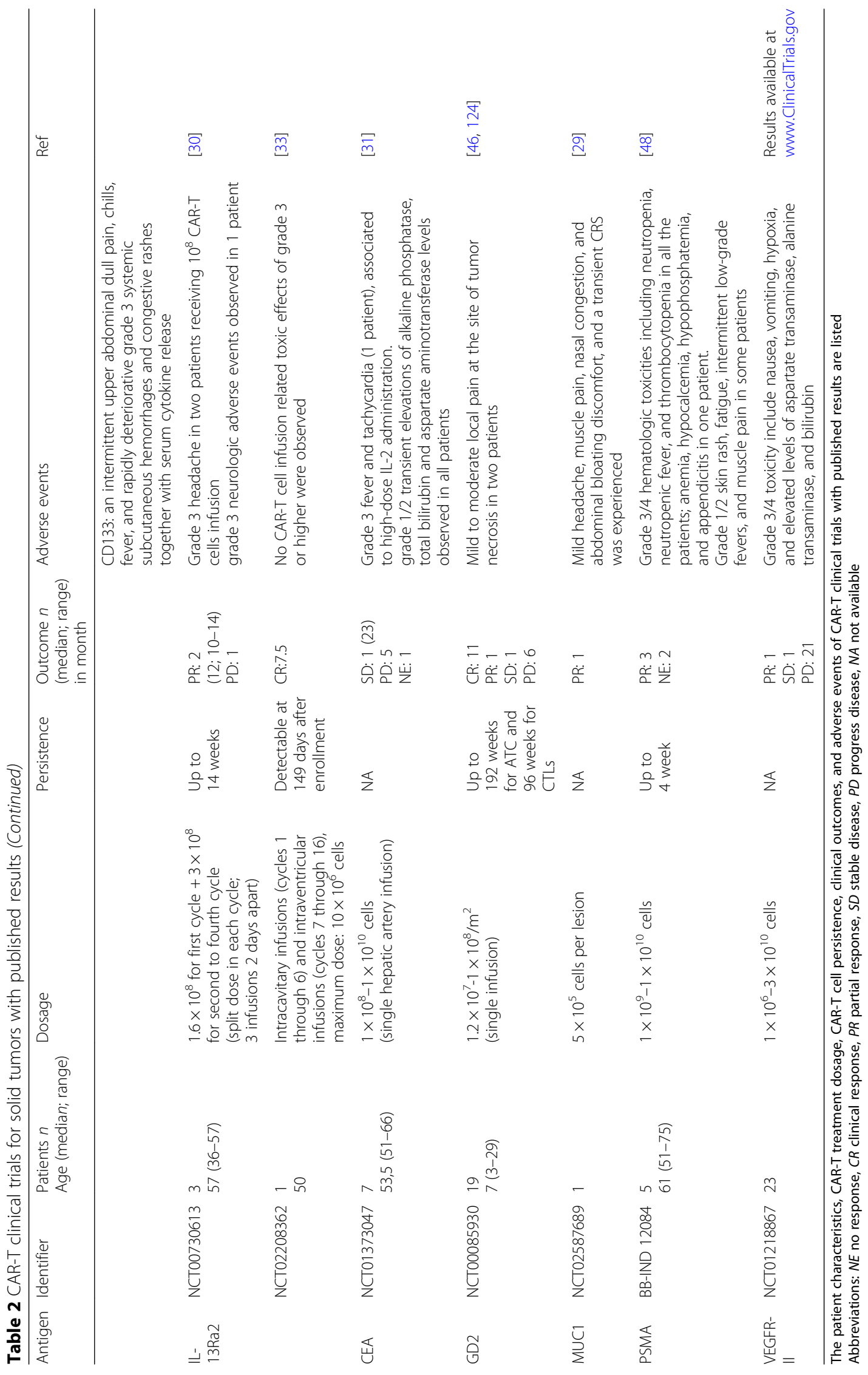




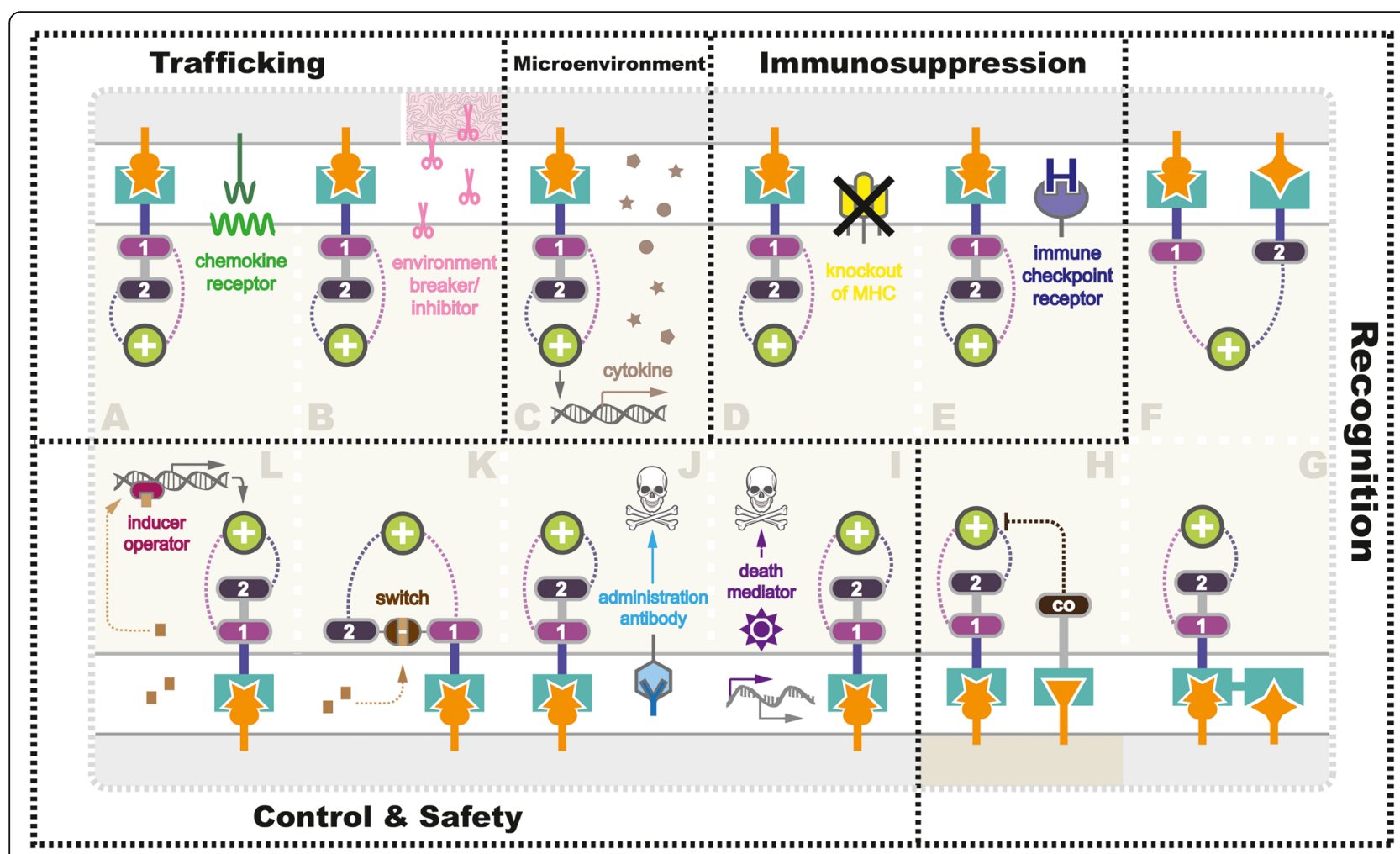

Fig. 3 Strategies being exploited to overcome challenges in CAR-T therapy in solid tumor. Various strategies are currently being tested in preclinical and clinical studies to overcome the challenges facing CAR-T therapy for solid tumor (gray), including $\mathbf{a}, \mathbf{b}$ enhancing T cell (beige) trafficking, $\mathbf{c}$ reforming tumor microenvironment (pink represented physiological barriers), $\mathbf{d}$, e anti-immunosuppression, elevating antigen recognition towards tumor (f, $\mathbf{g})$, and healthy cells (darker beige) (h), as well as i-I improving safety control using suicide switch or on-switch. Detailed mechanisms were further illustrated in the section "Overcoming Challenges with Smarter CAR Designs"

migratory capacity of CAR-T cells. Qin et al. generated two versions of CAR vectors, with or without a hinge domain, targeting hematopoietic, and solid tumor antigens [63]. The CARs with a hinge domain demonstrated better expansion and migration capacity in vitro and CAR-T cells expressing anti-mesothelin CARs containing a hinge domain showed enhanced antitumor activities.

\section{Reforming microenvironment}

Malignant transformation and the growth of tumor mass influence surrounding microenvironment, which subsequently gives rise to peripheral immune tolerance [64]. In return, tumor microenvironment provides a driving force contributing to not only tumor invasion and metastasis but also pharmacokinetic and pharmacodynamics resistance [65].

In studies targeting solid tumors, CAR-T cells surmount challenges conferred by anti-inflammatory factors (e.g., TGF- $\beta$ and IL-10), immune suppressor cells (Tregs, Bregs, myeloid cells, neutrophils, macrophages), antigen loss, tissue-specific alterations, etc., [51, 64]. Even if CAR-T cells successfully trafficked to the tumor sites, tumor microenvironment might suppress or even inactivate them. Therefore, with the aim to equip CAR$\mathrm{T}$ cells with capabilities to remodel the suppressive tumor microenvironment by secreting anti-cancer cytokines, strategies named TRUCK have been extensively utilized in CAR-T studies (Fig. 3c). To date, several cytokines have been adopted into TRUCK designs, including IL-12 [66, 67], IL-18 [68], and TNFRSF14 [69]. Recently, a leading-edge TRUCK system using synNotch receptor was described by Roybal et al. [70, 71], which was engineered to produce a range of specific payloads in response to the target antigens. In addition to inflammatory cytokines, the flexible synNotch system could also express pro-tumor cytokine antibodies, checkpoint antibodies, bispecific antibodies, and adjuvants. Using synNotch TRUCK system, more antitumor cytokines or other factors with the potential to reform the local environment based on the specific tumor heterogeneity might be exploited. For instance, reported as potential anticancer therapeutic candidates [72], cytokines such as IL24 should be further tested.

\section{Anti-immunosuppression}

Immunosuppression is a great challenge to effective CAR-T therapy, as it enables the tumor cell to escape 
from antitumor immune responses [73]. Various types of strategy have been exploited to engineer CAR-T cells to fight tumor immunosuppression. Manipulation of the endogenous TCR/MHC (Fig. 3d) and induction of additional immune checkpoint receptors (Fig. 3e) are two promising strategies investigated extensively.

Immune escape mediated by inhibitory pathways via the interaction of activated killer $\mathrm{T}$ cell receptors with their ligands, such as programmed cell death1 (PD-1) [74], and cytotoxic T lymphocyte antigen 4 (CTLA-4) on $\mathrm{T}$ cells is another major factor [75]. To overcome repressive solid tumor environments and enhance the activity and persistence of CAR-T cells, combined therapies using co-administration of immune checkpoint inhibitors or cytokines with CAR-T cells have been employed. Immune checkpoint inhibitors block the immune checkpoint pathways by targeting key regulators in the pathways (e.g., PD-1/PD-L1, CTLA4) to enhance the immune activity of patients' effector T cells [76]. The addition of anti-PD1 monoclonal antibody has been shown to mute the inhibitory effect of such receptors and enhance the function of CAR-T cells in preclinical models [77-79]. Many groups are now attempting to generate CAR-T cells resistant to PD1-PDL1 and CTLA4-CD80/CD86 signaling [80], and some of these CAR constructs are currently under investigation in clinical trials as discussed in the previous section (Table 1).

Immunosuppressive soluble factors, like TGF- $\beta$ and IL10, have been demonstrated to inhibit CAR-T cell activities [81]. Specifically, TGF- $\beta$ has direct negative effects on $\mathrm{T}$ cell differentiation and cytotoxic function, thus hampering $\mathrm{T}$ cell effector functions [82]. TGF- $\beta$ and IL10 can also inhibit antigen-presenting cells, leading to hampered activation of tumor-reactive $\mathrm{T}$ cells [83]. Other factors including prostaglandin E2 (PGE2) and adenosine have also been demonstrated to inhibit $\mathrm{T}$ cell proliferation and differentiation via signaling through G-coupled receptors [84, 85]. CAR-T cells can be potentially engineered to include the expression of a dominant-negative form of the receptor of these factors to overcome their inhibitory effects [86].

In addition to the soluble immunosuppressive factors, various suppressive surveilling immune cells within the tumor microenvironment, such as Tregs, myeloid-derived suppressor cell (MDSC), and tumor-associated macrophage (TAM)/tumor-associated neutrophils (TAN) with the so-called M2 and N2 phenotype, present another obstacle against successful CAR-T treatment. Tregs have been shown to inhibit $\mathrm{T}$ cell activity through cell-to-cell contact inhibition and via soluble factors such as TGF- $\beta$ and IL10 [87]. Tregs hamper $\mathrm{T}$ cell activity by producing TGF- $\beta$, IL10, and also other suppressive agents like IL35 and adenosine [88]. MDSC, M2-TAM, and N2-TAN inhibit antitumor immune response by producing TGF- $\beta$, PGE2, reactive oxygen/nitrogen species, and arginase $[89,90]$. M2-TAM can express high levels of PD-L1, which can interact with PD1 on CAR-T cells and inhibit them [91]. To overcome the effect of such suppressive surveilling immune cells, the CAR-T design has been proposed to target both the tumor cells and immune cells. Ruella et al. demonstrated the feasibility of targeting CD123-positive Hodgkin lymphoma cells and TAM [92]. However, whether this strategy is universally applicable to other malignancies, especially for the solid tumor, still needs to be further tested.

\section{Elevating recognition specificity}

The genetic heterogeneity of solid tumors is a major issue to treat such malignancies with CAR-T. Unlike CD19 in B cell leukemia, there is no such "panacea" antigen available for solid tumors. Worse still, crossreactions (i.e., "OFF target" and "ON target OFF tumor") with bystander non-tumor cells have been widely observed in CAR-T studies, leading to severe or even lethal adverse effects caused by $\mathrm{T}$ cells attack to the essential tissues.

This off-tumor toxicity could be restrained by designing CAR-T cells with enhanced specificity using two or more extracellular antigen recognition motifs. Presently, three major classes of bispecific CARs have been employed in $\mathrm{T}$ cell engineering, namely, dual CAR (Fig. 3f), tandem CAR (TanCAR) (Fig. 3g), and inhibitory CAR (iCAR) (Fig. 3h). Kloss et al. firstly reported co-expression of a suboptimal CAR with an additional chimeric costimulatory receptor (CCR), which sufficiently recognized and killed cells expressing the two antigens [93]. Dual T cells would be fully activated only when the two target antigens present simultaneously on tumor cell surface, which significantly strengthened the specificity and thus leaving the bystander cell untouched. Such dual CAR design was reported to generate specific toxicity towards tumor cells [94]. Recently, an advanced AND-gate circuit using synNotch receptors further upgraded the dual CAR system [70, 95]. Upon activation by associating with the first antigen, synNotch system induced a secondary CAR expression via intracellular transcriptional domain to modulate $\mathrm{T}$ cell activity in the presence of the second antigen. Interestingly, initially designed as an AND-gate circuit, a TanCAR that linked two distinct scFVs was adopted in treatments against HER2-positive glioblastoma [96, 97], yet has been reported to function as an OR-gate circuit [98], which could kill either CD19-positive or CD20positive leukemic cells in vivo. This TanCAR has been proved to be particularly useful in the clinic to prevent resistance caused by loss of antigens. To further 
minimize the $\mathrm{T}$ cell "on-target off-tumor" activity towards normal tissues, a NOT-gate circuit using a killing CAR and an inhibitory CAR was developed [99].

\section{Improving safety and control}

Most of the efforts at CAR-T engineering has been devoted to improve the strength of clinical response and to prolong $\mathrm{T}$ cell proliferation and persistence. However, the adverse effects observed in human trials suggest the urgency of additional consideration on safety control mechanisms. Utilization of control systems should be regarded as the priority in the next generation CAR- $\mathrm{T}$ therapy design as $\mathrm{T}$ cells are autonomous without regulatory mechanisms.

To date, a growing number of user-control systems have been developed to modulate CAR-T cell expression. In 1997, Bonini et al. firstly reported successful control of graft-versus-host disease (GVHD) using a suicide gene named herpes simplex virus thymidine kinase (HSV-tk) in adoptive $\mathrm{T}$ cell therapy, which rendered the $\mathrm{T}$ cells susceptible to ganciclovir treatment [100]. In 2001, Fas intracellular domain ( $\Delta$ Fas) was applied as a $T$ cell suicide switch to combat GVHD in marrow transplantation [101]. In 2011, Di Stasi and colleagues introduced a human caspase-9 (iCasp9) as an off-switch to conditionally trigger apoptosis of CAR-T cells by dimerization upon the treatment of small molecules (Fig. 3i) [102]. In hematopoietic stem cell transplantation recipients, $90 \%$ of iCasp9-modified $\mathrm{T}$ cells could be eliminated within 30 min under AP1903 administration without severe adverse effects or recurrence [103]. Synthetic death control switches are likely to be extensively utilized in future adoptive immunotherapy owing to their high effectiveness, easy controllability, short time of on-set, and mild adverse effects, despite that minority of the T cell populations could escape from the apoptotic signal, resulting in persistent cytotoxicity [103]. Alternatively, CAR-T cells could be removed via apoptosis using antigenspecific monoclonal antibodies (Fig. 3j), such as rituximab for CD20 epitope [104], and cetuximab for EGFR epitope [105]. In addition to selective elimination, expression of previously known antigens enables tracking of CAR-T cells in vivo.

In contrast to the death switch, a distinct class of oncontrol mechanisms is considered as safer manipulating strategies, as CAR-T cells using on-switches are defaulted to be unresponsive. In this design, CARs are conditionally expressed or form a functional structure in the presence of the inducer, removal of which terminates the transcription or inactivates CARs. Wu et al. described an advanced on-switch CAR using a split construct in vivo (Fig. 3k). The split CAR design distributed into two separate polypeptides, an extracellular binding domain $\mathrm{scFv}$ and a downstream signaling element
ITAM, two parts reassembled and activated under the treatment of a small molecule rapalog [106]. In 2016, Morsut and colleagues further developed a tissue ligandspecific on-switch CAR construct based on synNotch receptors using a tetracycline (Tet)-regulated promoter (Fig. 3l) [107]. It is noteworthy that expression levels of CARs are generally dose-dependent before saturation, which allows manipulating the strength of CAR expression accordingly. In Tet-on systems, CARs still exist after removal of the inducer; hence, it requires further depletion to eliminate CAR-T cells ultimately. At this stage, an antibody like myc could be conveniently applied to target the tagged CARs as a safeguard factors after adoptive transfer [108]. In principle, integration of the on- and off-switch systems enables us to turn on the expression of CARs at the wanted time point, to turn off when the doses are sufficient, and to erase $\mathrm{T}$ cells from patients once the treatments are completed.

Strategies involving codon optimization [109-111] or construct alteration using lentivirus [112], transposon [113], or RNA [114] were also employed in the next generation CAR design to ameliorate side effects resulted from $\mathrm{T}$ cell overactivation. Hopefully, a combination of suicide/on switches, optimized coding, relevant downstream feedback response, and sensor circuits in the future trials could help to establish global control systems to modulate the expression, strength, and timing of the engineered CAR-T cells.

\section{Conclusion}

CAR-T has demonstrated itself as a promising treatment for the solid tumor in preclinical and clinical studies. Progress in the following aspects of CAR-T should facilitate the development of the therapy. First, CAR designs need to be further optimized to give better $\mathrm{T}$ cell activation, recognition specificity, antitumor activity, and safety control. The search for optimal signaling and costimulatory domains will continue to improve the efficacy of CAR-T therapy. Application of bispecific CAR is a promising way to enhance the tumor cell recognition specificity, limiting unexpected attack to the normal cells. Because of the genetic heterogeneity, personalized modification during CAR construction might be needed to deliver the maximum antitumor effect. Second, identification of the most suitable $\mathrm{T}$ cell subset for genetic engineering and the establishment of a standard ex vivo $\mathrm{T}$ cell processing procedure are critical for producing long-lasting CAR-T cell persistence and memory for optimized antitumor response. Additional modifications to CAR-modified $\mathrm{T}$ cells might be necessary to overcome immunosuppressive tumor microenvironments. Strategies like the introduction of anti-cancer cytokines (IL12), manipulation of the immune checkpoint signaling (PD1/CTLA4), immunosuppressive soluble factors 
(TGF- $\beta$, IL10), and suppressive surveilling immune cells are aspects worth to be explored. Third, the establishment of standard clinical protocols is needed. Improvements in the CAR-T cell itself will require parallel developments in clinical protocol design, including patient preconditioning, cytokine support, and other potential co-administered treatments. Preconditioning of the patient before adoptive cell therapy is thought to have a significant effect on the immune response, thereby producing a potential therapeutic window for CAR-T cell activity. Improvements in CAR design and better understating of the interaction between tumor and immune system will help to overcome the hurdles currently limiting the application CAR-T in solid tumor treatment.

\section{Abbreviations}

ALL: Acute lymphoblastic leukemia; BTC: Biliary tract cancer;

CAIX: Carboxyanhydrase-IX; CARs: Chimeric antigen receptors; CAR-T cells: Chimeric antigen receptor T cells; CCA: Cholangiocarcinoma;

CCR: Chimeric costimulatory receptor; CEA: Carcinoembryonic antigen; CMET: Hepatocyte growth factor receptor; CTLA-4: Cytotoxic T-lymphocyte antigen 4; EGFR: Epidermal growth factor receptor; EpCAM: Epithelial cell adhesion molecule; EphA2: EPH receptor A2; FAP: Fibroblast activation protein a; FDA: Food and Drug Administration; FR: Folate receptor; GD2: Disialoganglioside; GPC3: Glypican-3; GVHD: Graft-versus-host disease; HER2: Human epidermal growth factor receptor-2; HSV-tk: Herpes simplex virus thymidine kinase; iCAR: Inhibitory CAR; IL: Interleukin; L1-CAM: L1 cell adhesion molecule; MDSC: Myeloid-derived suppressor cell; MPM: Malignant pleural mesothelioma; MSLN: Mesothelin; MUC1: Mucin; NK cells: Natural killer cells; NSCLC: Non-small-cell lung carcinoma; PD-1: Programmed cell death-1; PDAC: Pancreatic ductal adenocarcinoma; PGE2: Prostaglandin E2; PR: Partial response; PSMA: Prostate-specific membrane antigen; ROR1: Receptor tyrosine kinase-like orphan receptor 1; scFV: Single chain variable fragments; TAA: Tumor-associated antigens; TAM: Tumor-associated macrophage; TAN: Tumor-associated neutrophils; TCR: T cell receptor; Tet: Tetracycline; Treg cells: Regulatory T cells; TRUCK: T cell redirected for universal cytokine-mediated killing; VEGFR: Vascular endothelial growth factor receptor

\section{Acknowledgements}

We apologize for not being able to cite all the related work due to page limit.

\section{Funding}

This work was supported by the National Natural Science Foundation of China (no. 31500657) and the International Collaboration Projects from the Science and Technology Bureau of Sichuan Province (no. 2017HH0097) to Jian Li. Wenwen Li was supported by the China Scholarship Council.

\section{Availability of data and materials}

Data sharing is not applicable to this article as no datasets were generated or analyzed during the current study.

\section{Authors' contributions}

$J L, W L, G K$, and QZ conceived the study and wrote the manuscript. JL, WL, $\mathrm{KH}$, and $\mathrm{YZ}$ performed the literature search. All authors read and approved the final manuscript

\section{Ethics approval and consent to participate}

Not applicable.

\section{Consent for publication}

All authors have read and approved the final manuscript for publication.

\section{Competing interests}

The authors declare that they have no competing interests.

\section{Publisher's Note}

Springer Nature remains neutral with regard to jurisdictional claims in published maps and institutional affiliations.

\section{Author details}

${ }^{1}$ School of Medicine, Chengdu University, Chengdu 610106, China. ${ }^{2}$ UCL Institute of Ophthalmology, 11-43 Bath Street, London EC1V 9EL, UK. ${ }^{3}$ Sichuan Industrial Institute of Antibiotics, Chengdu University, Chengdu 610052, China. ${ }^{4}$ Section of Hematology-Oncology, Department of Pediatrics, Yale University School of Medicine, New Haven, CT 06520, USA. ${ }^{5}$ College of Pharmacy and Biological Engineering, Chengdu University, Chengdu 610106, China

Received: 1 December 2017 Accepted: 6 February 2018

Published online: 13 February 2018

\section{References}

1. Panel OKs CAR T therapy for leukemia. Cancer Discov. 2017;7(9):924.

2. Grupp SA, et al. Chimeric antigen receptor-modified T cells for acute lymphoid leukemia. N Engl J Med. 2013;368(16):1509-18.

3. Gross G, Waks T, Eshhar Z. Expression of immunoglobulin-T-cell receptor chimeric molecules as functional receptors with antibody-type specificity. Proc Natl Acad Sci U S A. 1989;86(24):10024-8.

4. Ahmad ZA, et al. scFv antibody: principles and clinical application. Clin Dev Immunol. 2012;2012:980250.

5. Dotti G, Savoldo B, Brenner M. Fifteen years of gene therapy based on chimeric antigen receptors: "are we nearly there yet?". Hum Gene Ther. 2009:20(11):1229-39.

6. Hombach A, et al. Human CD4+ T cells lyse target cells via granzyme/ perforin upon circumvention of MHC class II restriction by an antibody-like immunoreceptor. J Immunol. 2006:177(8):5668-75.

7. Fesnak $A D$, June $C H$, Levine BL. Engineered $T$ cells: the promise and challenges of cancer immunotherapy. Nat Rev Cancer. 2016;16(9):566-81.

8. Irving BA, Weiss A. The cytoplasmic domain of the T cell receptor zeta chain is sufficient to couple to receptor-associated signal transduction pathways. Cell. 1991;64(5):891-901.

9. Haynes NM, et al. Redirecting mouse CTL against colon carcinoma: superior signaling efficacy of single-chain variable domain chimeras containing TCRzeta vs Fc epsilon Rl-gamma. J Immunol. 2001:166(1):182-7.

10. Brocker T, Karjalainen K. Signals through T cell receptor-zeta chain alone are insufficient to prime resting T lymphocytes. J Exp Med. 1995;181(5):1653-9.

11. Finney HM, et al. Chimeric receptors providing both primary and costimulatory signaling in T cells from a single gene product. J Immunol. 1998;161(6):2791-7.

12. Shen $\mathrm{CJ}$, et al. Chimeric antigen receptor containing ICOS signaling domain mediates specific and efficient antitumor effect of T cells against EGFRvIll expressing glioma. J Hematol Oncol. 2013;6:33.

13. Maher J, et al. Human T-lymphocyte cytotoxicity and proliferation directed by a single chimeric TCRzeta /CD28 receptor. Nat Biotechnol. 2002;20(1):70-5.

14. Savoldo B, et al. CD28 costimulation improves expansion and persistence of chimeric antigen receptor-modified T cells in lymphoma patients. J Clin Invest. 2011;121(5):1822-6.

15. Kofler DM, et al. CD28 costimulation impairs the efficacy of a redirected tcell antitumor attack in the presence of regulatory $t$ cells which can be overcome by preventing Lck activation. Mol Ther. 2011;19(4):760-7.

16. Koehler $\mathrm{H}$, et al. CD28 costimulation overcomes transforming growth factorbeta-mediated repression of proliferation of redirected human CD4+ and CD8+ T cells in an antitumor cell attack. Cancer Res. 2007;67(5):2265-73.

17. Pule MA, et al. A chimeric $T$ cell antigen receptor that augments cytokine release and supports clonal expansion of primary human T cells. Mol Ther 2005;12(5):933-41.

18. Hombach AA, et al. OX40 costimulation by a chimeric antigen receptor abrogates CD28 and IL-2 induced IL-10 secretion by redirected CD4(+) T cells. Oncoimmunology. 2012;1 (4):458-66.

19. Chmielewski M, Hombach AA, Abken H. Of CARs and TRUCKs: chimeric antigen receptor (CAR) T cells engineered with an inducible cytokine to modulate the tumor stroma. Immunol Rev. 2014;257(1):83-90.

20. Chmielewski M, Abken H. TRUCKs: the fourth generation of CARs. Expert Opin Biol Ther. 2015;15(8):1145-54. 
21. Lopez-Albaitero A, et al. Overcoming resistance to HER2-targeted therapy with a novel HER2/CD3 bispecific antibody. Oncoimmunology. 2017;6(3):e1267891.

22. Yu S, et al. Chimeric antigen receptor $T$ cells: a novel therapy for solid tumors. J Hematol Oncol. 2017;10(1):78

23. Liu B, Song Y, Liu D. Clinical trials of CAR-T cells in China. J Hematol Oncol 2017;10(1):166

24. Hartmann J, et al. Clinical development of CAR T cells_challenges and opportunities in translating innovative treatment concepts. EMBO Mol Med. 2017;9(9):1183-97.

25. Gargett T, Brown MP. The inducible caspase-9 suicide gene system as a "safety switch" to limit on-target, off-tumor toxicities of chimeric antigen receptor T cells. Front Pharmacol. 2014,5:235.

26. Diaconu I, et al. Inducible caspase-9 selectively modulates the toxicities of CD19-specific chimeric antigen receptor-modified T cells. Mol Ther. 2017; 25(3):580-92.

27. Chen $\mathrm{N}$, et al. CAR T-cell intrinsic PD-1 checkpoint blockade: a two-in-one approach for solid tumor immunotherapy. Oncoimmunology. 2017;6(2): e1273302.

28. Ahmed N, et al. Human epidermal growth factor receptor 2 (HER2)-specific chimeric antigen receptor-modified T cells for the immunotherapy of HER2positive sarcoma. J Clin Oncol. 2015;33(15):1688-96.

29. You F, et al. Phase 1 clinical trial demonstrated that MUC1 positive metastatic seminal vesicle cancer can be effectively eradicated by modified anti-MUC1 chimeric antigen receptor transduced T cells. Sci China Life Sci. 2016:59(4):386-97.

30. Brown CE, et al. Bioactivity and safety of IL13Ralpha2-redirected chimeric antigen receptor CD8+ T cells in patients with recurrent glioblastoma. Clin Cancer Res. 2015;21(18):4062-72.

31. Katz SC, et al. Phase I hepatic immunotherapy for metastases study of intraarterial chimeric antigen receptor-modified T-cell therapy for CEA+ liver metastases. Clin Cancer Res. 2015;21(14):3149-59.

32. Petrausch $U$, et al. Re-directed $T$ cells for the treatment of fibroblast activation protein (FAP)-positive malignant pleural mesothelioma (FAPME-1). BMC Cancer. 2012;12:615

33. Brown $\mathrm{CE}$, et al. Regression of glioblastoma after chimeric antigen receptor T-cell therapy. N Engl J Med. 2016;375(26):2561-9.

34. Brentjens RJ, et al. Safety and persistence of adoptively transferred autologous CD19-targeted T cells in patients with relapsed or chemotherapy refractory B-cell leukemias. Blood. 2011;118(18):4817-28.

35. Kershaw $\mathrm{MH}$, et al. A phase I study on adoptive immunotherapy using gene-modified T cells for ovarian cancer. Clin Cancer Res. 2006;12(20 Pt 1): 6106-15.

36. Lamers $\mathrm{CH}$, et al. Treatment of metastatic renal cell carcinoma with CAIX CAR-engineered T cells: clinical evaluation and management of on-target toxicity. Mol Ther. 2013;21(4):904-12.

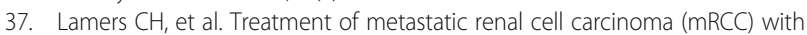
CAIX CAR-engineered T-cells--a completed study overview. Biochem Soc Trans. 2016;44(3):951-9.

38. Park JR, et al. Adoptive transfer of chimeric antigen receptor re-directed cytolytic T lymphocyte clones in patients with neuroblastoma. Mol Ther. 2007;15(4):825-33.

39. Beatty $\mathrm{GL}$, et al. Mesothelin-specific chimeric antigen receptor mRNAengineered T cells induce anti-tumor activity in solid malignancies. Cancer Immunol Res. 2014;2(2):112-20.

40. Maus MV, et al. T cells expressing chimeric antigen receptors can cause anaphylaxis in humans. Cancer Immunol Res. 2013;1(1):26-31.

41. Beatty $\mathrm{GL}$, et al. Safety and antitumor activity of chimeric antigen receptor modified T cells in patients with chemotherapy refractory metastatic pancreatic cancer. J Clin Oncol. 2015;33(15_suppl):3007.

42. Feng $\mathrm{K}$, et al. Phase I study of chimeric antigen receptor modified T cells in treating HER2-positive advanced biliary tract cancers and pancreatic cancers. Protein Cell. 2017. https://doi.org/10.1007/s13238-017-0440-4.

43. Ahmed N, et al. Autologous HER2 CMV bispecific CAR T cells are safe and demonstrate clinical benefit for glioblastoma in a phase I trial. J Immunother Cancer. 2015;3(Suppl 2):011.

44. Feng $\mathrm{K}$, et al. Chimeric antigen receptor-modified $T$ cells for the immunotherapy of patients with EGFR-expressing advanced relapsed/ refractory non-small cell lung cancer. Sci China Life Sci. 2016;59(5):468-79.

45. Feng KC, et al. Cocktail treatment with EGFR-specific and CD133-specific chimeric antigen receptor-modified T cells in a patient with advanced cholangiocarcinoma. J Hematol Oncol. 2017;10(1):4.
46. Louis CU, et al. Antitumor activity and long-term fate of chimeric antigen receptor-positive T cells in patients with neuroblastoma. Blood. 2011; 118(23):6050-6.

47. Tchou J, et al. Safety and efficacy of intratumoral injections of chimeric antigen receptor (CAR) T cells in metastatic breast cancer. Cancer Immunol Res. 2017;5(12):1152-61.

48. Junghans RP, et al. Phase I trial of anti-PSMA designer CAR-T cells in prostate cancer: possible role for interacting interleukin 2-T cell pharmacodynamics as a determinant of clinical response. Prostate. 2016; 76(14):1257-70

49. Hanahan D, Weinberg RA. Hallmarks of cancer: the next generation. Cell. 2011;144(5):646-74.

50. Stuelten $\mathrm{CH}$, et al. Breast cancer cells induce stromal fibroblasts to express MMP-9 via secretion of TNF-alpha and TGF-beta. J Cell Sci. 2005;118(Pt 10): 2143-53.

51. Palucka AK, Coussens LM. The basis of oncoimmunology. Cell. 2016;164(6): 1233-47.

52. Friedl $P$, Alexander $S$. Cancer invasion and the microenvironment: plasticity and reciprocity. Cell. 2011;147(5):992-1009.

53. Newick K, Moon E, Albelda SM. Chimeric antigen receptor T-cell therapy for solid tumors. Mol Ther Oncolytics. 2016:3:16006.

54. Moon EK, et al. Expression of a functional CCR2 receptor enhances tumor localization and tumor eradication by retargeted human $T$ cells expressing a mesothelin-specific chimeric antibody receptor. Clin Cancer Res. 2011;17(14): 4719-30.

55. Craddock JA, et al. Enhanced tumor trafficking of GD2 chimeric antigen receptor T cells by expression of the chemokine receptor CCR2b. J Immunother. 2010:33(8):780-8.

56. Schuberth PC, et al. Treatment of malignant pleural mesothelioma by fibroblast activation protein-specific re-directed T cells. J Transl Med. 2013; 11:187.

57. Lo A, et al. Tumor-promoting desmoplasia is disrupted by depleting FAPexpressing stromal cells. Cancer Res. 2015;75(14):2800-10.

58. Chinnasamy D, et al. Gene therapy using genetically modified lymphocytes targeting VEGFR-2 inhibits the growth of vascularized syngenic tumors in mice. J Clin Invest. 2010;120(11):3953-68.

59. Caruana I, et al. Heparanase promotes tumor infiltration and antitumor activity of CAR-redirected T lymphocytes. Nat Med. 2015;21(5):524-9.

60. Garin-Chesa P, Old LJ, Rettig WJ. Cell surface glycoprotein of reactive stromal fibroblasts as a potential antibody target in human epithelial cancers. Proc Natl Acad Sci U S A. 1990;87(18):7235-9.

61. Beyer I, van Rensburg R, Lieber A. Overcoming physical barriers in cancer therapy. Tissue Barriers. 2013;1(1):e23647.

62. Beyer I, et al. Controlled extracellular matrix degradation in breast cancer tumors improves therapy by trastuzumab. Mol Ther. 2011;19(3):479-89.

63. Qin $L$, et al. Incorporation of a hinge domain improves the expansion of chimeric antigen receptor T cells. J Hematol Oncol. 2017;10(1):68.

64. Balkwill FR, Capasso M, Hagemann T. The tumor microenvironment at a glance. J Cell Sci. 2012;125(Pt 23):5591-6.

65. Khawar IA, Kim JH, Kuh HJ. Improving drug delivery to solid tumors: priming the tumor microenvironment. J Control Release. 2015;201:78-89.

66. Kerkar SP, et al. Tumor-specific CD8+ T cells expressing interleukin-12 eradicate established cancers in lymphodepleted hosts. Cancer Res. 2010; 70(17):6725-34

67. Pegram HJ, et al. Tumor-targeted T cells modified to secrete IL-12 eradicate systemic tumors without need for prior conditioning. Blood. 2012;119(18): 4133-41.

68. Avanzi MP, et al. IL-18 secreting CAR T cells enhance cell persistence, induce prolonged B cell aplasia and eradicate CD19+ tumor cells without need for prior conditioning. Blood. 2016;128(22):816.

69. Boice $M$, et al. Loss of the HVEM tumor suppressor in lymphoma and restoration by modified CAR-T cells. Cell. 2016;167(2):405-18. e13

70. Roybal KT, et al. Engineering T cells with customized therapeutic response programs using synthetic notch receptors. Cell. 2016;167(2):419-32. e16

71. Roybal KT, Lim WA. Synthetic immunology: hacking immune cells to expand their therapeutic capabilities. Annu Rev Immunol. 2017;35:229-53.

72. Dent $P$, et al. MDA-7/IL-24 as a cancer therapeutic: from bench to bedside Anti-Cancer Drugs. 2010;21(8):725-31.

73. Beatty $\mathrm{GL}$, Moon EK. Chimeric antigen receptor $\mathrm{T}$ cells are vulnerable to immunosuppressive mechanisms present within the tumo microenvironment. Oncoimmunology. 2014;3(11):e970027. 
74. Chevolet I, et al. Characterization of the in vivo immune network of IDO, tryptophan metabolism, PD-L1, and CTLA-4 in circulating immune cells in melanoma. Oncoimmunology. 2015;4(3):e982382.

75. Ewing MM, et al. T-cell co-stimulation by CD28-CD80/86 and its negative regulator CTLA-4 strongly influence accelerated atherosclerosis development. Int J Cardiol. 2013;168(3):1965-74.

76. Hegde UP, Mukherji B. Current status of chimeric antigen receptor engineered T cell-based and immune checkpoint blockade-based cancer immunotherapies. Cancer Immunol Immunother. 2017;66(9):1113-21.

77. Condomines $M$, et al. Tumor-targeted human T cells expressing CD28based chimeric antigen receptors circumvent CTLA-4 inhibition. PLoS One. 2015;10(6):e0130518.

78. Prosser ME, et al. Tumor PD-L1 co-stimulates primary human CD8(+) cytotoxic T cells modified to express a PD1:CD28 chimeric receptor. Mol Immunol. 2012:51(3-4):263-72.

79. John LB, et al. Anti-PD-1 antibody therapy potently enhances the eradication of established tumors by gene-modified T cells. Clin Cancer Res. 2013;19(20):5636-46.

80. Pegram HJ, Park JH, Brentjens RJ. CD28z CARs and armored CARs. Cancer J. 2014;20(2):127-33.

81. Pardoll DM. The blockade of immune checkpoints in cancer immunotherapy. Nat Rev Cancer. 2012;12(4):252-64.

82. Massague J. TGFbeta in cancer. Cell. 2008;134(2):215-30.

83. Enblad G, Karlsson H, Loskog AS. CAR T-cell therapy: the role of physical barriers and immunosuppression in lymphoma. Hum Gene Ther. 2015;26(8):498-505.

84. Newick K, et al. Augmentation of CAR T-cell trafficking and antitumor efficacy by blocking protein kinase a localization. Cancer Immunol Res. 2016:4(6):541-51.

85. Su Y, et al. Cooperation of adenosine and prostaglandin E2 (PGE2) in amplification of cAMP-PKA signaling and immunosuppression. Cancer Immunol Immunother. 2008;57(11):1611-23.

86. Foster $A E$, et al. Antitumor activity of EBV-specific $T$ lymphocytes transduced with a dominant negative TGF-beta receptor. J Immunother. 2008;31(5): 500-5.

87. Sakaguchi $\mathrm{S}$, et al. FOXP3+ regulatory $T$ cells in the human immune system. Nat Rev Immunol. 2010;10(7):490-500

88. Roychoudhuri R, Eil RL, Restifo NP. The interplay of effector and regulatory $T$ cells in cancer. Curr Opin Immunol. 2015;33:101-11.

89. Gabrilovich DI, Nagaraj S. Myeloid-derived suppressor cells as regulators of the immune system. Nat Rev Immunol. 2009;9(3):162-74.

90. Mussai F, et al. Neuroblastoma arginase activity creates an immunosuppressive microenvironment that impairs autologous and engineered immunity. Cancer Res. 2015;75(15):3043-53.

91. Ostuni R, et al. Macrophages and cancer: from mechanisms to therapeutic implications. Trends Immunol. 2015;36(4):229-39.

92. Ruella $\mathrm{M}$, et al. Overcoming the immunosuppressive tumor microenvironment of Hodgkin lymphoma using chimeric antigen receptor T cells. Cancer Discov. 2017;7(10):1154-67.

93. Kloss CC, et al. Combinatorial antigen recognition with balanced signaling promotes selective tumor eradication by engineered T cells. Nat Biotechnol. 2013;31(1):71-5.

94. Wilkie $\mathrm{S}$, et al. Dual targeting of ErbB2 and MUC1 in breast cancer using chimeric antigen receptors engineered to provide complementary signaling. J Clin Immunol. 2012;32(5):1059-70.

95. Roybal KT, et al. Precision tumor recognition by $T$ cells with combinatorial antigen-sensing circuits. Cell. 2016;164(4):770-9.

96. Grada Z, et al. TanCAR: a novel bispecific chimeric antigen receptor for cancer immunotherapy. Mol Ther Nucleic Acids. 2013;2:e105.

97. Hegde $\mathrm{M}$, et al. A bispecific chimeric antigen receptor molecule enhances $T$ cell activation through dual immunological synapse formation and offsets antigen escape in glioblastoma. J Immunother Cancer. 2015;3(Suppl 2):O3.

98. Zah E, et al. T cells expressing CD19/CD20 bispecific chimeric antigen receptors prevent antigen escape by malignant B cells. Cancer Immunol Res. 2016;4(6):498-508

99. Fedorov VD, Themeli M, Sadelain M. PD-1- and CTLA-4-based inhibitory chimeric antigen receptors (iCARs) divert off-target immunotherapy responses. Sci Transl Med. 2013;5(215):215ra172.

100. Bonini C, et al. HSV-TK gene transfer into donor lymphocytes for control of allogeneic graft-versus-leukemia. Science. 1997;276(5319):1719-24.

101. Thomis DC, et al. A Fas-based suicide switch in human T cells for the treatment of graft-versus-host disease. Blood. 2001;97(5):1249-57.
102. Di Stasi A, et al. Inducible apoptosis as a safety switch for adoptive cell therapy. N Engl J Med. 2011;365(18):1673-83.

103. Zhou $X$, et al. Long-term outcome after haploidentical stem cell transplant and infusion of T cells expressing the inducible caspase 9 safety transgene. Blood. 2014;123(25):3895-905.

104. Vogler l, et al. An improved bicistronic CD20/tCD34 vector for efficient purification and in vivo depletion of gene-modified $T$ cells for adoptive immunotherapy. Mol Ther. 2010;18(7):1330-8.

105. Wang $X$, et al. A transgene-encoded cell surface polypeptide for selection, in vivo tracking, and ablation of engineered cells. Blood. 2011;118(5):1255-63.

106. Wu CY, et al. Remote control of therapeutic $T$ cells through a small molecule-gated chimeric receptor. Science. 2015;350(6258):aab4077.

107. Morsut $L$, et al. Engineering customized cell sensing and response behaviors using synthetic notch receptors. Cell. 2016;164(4):780-91.

108. Kieback $E$, et al. A safeguard eliminates $T$ cell receptor gene-modified autoreactive $T$ cells after adoptive transfer. Proc Natl Acad Sci U S A. 2008;105(2):623-8.

109. Kuball J, et al. Facilitating matched pairing and expression of TCR chains introduced into human T cells. Blood. 2007;109(6):2331-8.

110. Cohen CJ, et al. Enhanced antitumor activity of $\mathrm{T}$ cells engineered to express T-cell receptors with a second disulfide bond. Cancer Res. 2007; 67(8):3898-903.

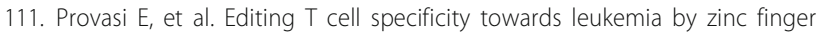
nucleases and lentiviral gene transfer. Nat Med. 2012;18(5):807-15.

112. Porter $\mathrm{DL}$, et al. Chimeric antigen receptor-modified $\mathrm{T}$ cells in chronic lymphoid leukemia. N Engl J Med. 2011;365(8):725-33.

113. Jin $Z$, et al. The hyperactive sleeping beauty transposase SB100X improves the genetic modification of $T$ cells to express a chimeric antigen receptor. Gene Ther. 2011;18(9):849-56.

114. Singh N, Barrett DM, Grupp SA. Roadblocks to success for RNA CARs in solid tumors. Oncoimmunology. 2014;3(12):e962974.

115. Lamers $\mathrm{CH}$, et al. Gene-modified T cells for adoptive immunotherapy of renal cell cancer maintain transgene-specific immune functions in vivo. Cancer Immunol Immunother. 2007;56(12):1875-83.

116. Lamers $\mathrm{CH}$, et al. Immune responses to transgene and retroviral vector in patients treated with ex vivo-engineered T cells. Blood. 2011;117(1): 72-82.

117. Lamers $\mathrm{CH}$, et al. Treatment of metastatic renal cell carcinoma with autologous T-lymphocytes genetically retargeted against carbonic anhydrase IX: first clinical experience. J Clin Oncol. 2006;24(13):e20-2.

118. Abate-Daga $D$, et al. A novel chimeric antigen receptor against prostate stem cell antigen mediates tumor destruction in a humanized mouse model of pancreatic cancer. Hum Gene Ther. 2014;25(12):1003-12.

119. Morgan RA, et al. Case report of a serious adverse event following the administration of $\mathrm{T}$ cells transduced with a chimeric antigen receptor recognizing ERBB2. Mol Ther. 2010;18(4):843-51.

120. Morgan RA, et al. Recognition of glioma stem cells by genetically modified T cells targeting EGFRvIll and development of adoptive cell therapy for glioma. Hum Gene Ther. 2012;23(10):1043-53.

121. Saied A, et al. Neutrophil:lymphocyte ratios and serum cytokine changes after hepatic artery chimeric antigen receptor-modified T-cell infusions for liver metastases. Cancer Gene Ther. 2014;21(11):457-62.

122. Katz SC, et al. Regional CAR-T cell infusions for peritoneal carcinomatosis are superior to systemic delivery. Cancer Gene Ther. 2016;23(5):142-8.

123. Golubovskaya $V$, et al. CD47-CAR-T cells effectively kill target cancer cells and block pancreatic tumor growth. Cancers (Basel). 2017;9(10):139.

124. Pule MA, et al. Virus-specific T cells engineered to coexpress tumor-specific receptors: persistence and antitumor activity in individuals with neuroblastoma. Nat Med. 2008;14(11):1264-70.

125. Heczey A, et al. Invariant NKT cells with chimeric antigen receptor provide a novel platform for safe and effective cancer immunotherapy. Blood. 2014; 124(18):2824-33.

126. Tanaka $M$, et al. Vaccination targeting native receptors to enhance the function and proliferation of chimeric antigen receptor (CAR)-modified T cells. Clin Cancer Res. 2017;23(14):3499-509.

127. Stroncek DF, et al. Elutriated lymphocytes for manufacturing chimeric antigen receptor T cells. J Transl Med. 2017;15(1):59.

128. Zuccolotto G, et al. PSMA-specific CAR-engineered T cells eradicate disseminated prostate cancer in preclinical models. PLoS One. 2014;9(10): e109427. 
129. Gohil SH, et al. An ROR1 bi-specific T-cell engager provides effective targeting and cytotoxicity against a range of solid tumors. Oncoimmunology. 2017;6(7):e1326437.

130. Koneru $\mathrm{M}$, et al. A phase I clinical trial of adoptive T cell therapy using IL-12 secreting MUC-16(ecto) directed chimeric antigen receptors for recurrent ovarian cancer. J Transl Med. 2015;13:102.

Submit your next manuscript to BioMed Central and we will help you at every step:

- We accept pre-submission inquiries

- Our selector tool helps you to find the most relevant journal

- We provide round the clock customer support

- Convenient online submission

- Thorough peer review

- Inclusion in PubMed and all major indexing services

- Maximum visibility for your research

Submit your manuscript at www.biomedcentral.com/submit 